\title{
RESEARCH
}

Open Access

\section{Using psychological theory to inform methods to optimize the implementation of a hand hygiene intervention}

Veronique M Boscart ${ }^{1,2}$, Geoff R Fernie ${ }^{2,3}$, Jae H Lee ${ }^{2}$ and Susan B Jaglal ${ }^{2,4^{*}}$

\begin{abstract}
Background: Careful hand hygiene $(\mathrm{HH})$ is the single most important factor in preventing the transmission of infections to patients, but compliance is difficult to achieve and maintain. A lack of understanding of the processes involved in changing staff behaviour may contribute to the failure to achieve success. The purpose of this study was to identify nurses' and administrators' perceived barriers and facilitators to current $\mathrm{HH}$ practices and the implementation of a new electronic monitoring technology for $\mathrm{HH}$.
\end{abstract}

Methods: Ten key informant interviews (three administrators and seven nurses) were conducted to explore barriers and facilitators related to $\mathrm{HH}$ and the impact of the new technology on outcomes. The semi structured interviews were based on the Theoretical Domains Framework by Michie et al. and conducted prior to intervention implementation. Data were explored using an inductive qualitative analysis approach. Data between administrators and nurses were compared.

Results: In 9 of the 12 domains, nurses and administrators differed in their responses. Administrators believed that nurses have insufficient knowledge and skills to perform $\mathrm{HH}$, whereas the nurses were confident they had the required knowledge and skills. Nurses focused on immediate consequences, whereas administrators highlighted long-term outcomes of the system. Nurses concentrated foremost on their personal safety and their families' safety as a source of motivation to perform $\mathrm{HH}$, whereas administrators identified professional commitment, incentives, and goal setting. Administrators stated that the staff do not have the decision processes in place to judge whether $\mathrm{HH}$ is necessary or not. They also highlighted the positive aspects of teams as a social influence, whereas nurses were not interested in group conformity or being compared to others. Nurses described the importance of individual feedback and self-monitoring in order to increase their performance, whereas administrators reported different views.

Conclusions: This study highlights the benefits of using a structured approach based on psychological theory to inform an implementation plan for a behavior change intervention. This work is an essential step towards systematically identifying factors affecting nurses' behaviour associated with $\mathrm{HH}$.

Keywords: Hand hygiene, Knowledge translation, Compliance, Behaviour change, Electronic monitoring

\footnotetext{
* Correspondence: susan.jaglal@utoronto.ca

${ }^{2}$ Toronto Rehabilitation Institute, Toronto, ON, Canada

${ }^{4}$ Department of Physical Therapy, University of Toronto, Toronto, ON, Canada

Full list of author information is available at the end of the article
} 


\section{Background}

Transmission of infections within healthcare institutions presents a significant threat to the health of patients and staff [1]. It has been estimated that there are 2 million hospital-acquired infections (HAIs) per year globally, affecting $10 \%$ of hospitalized patients [2,3]. In the United States, HAIs are estimated to cause 90,000 deaths annually and result in $\$ 5.7$ billion in excess healthcare costs $[4,5]$. In Canada, HAIs affect 220,000 patients, resulting in 8,000 deaths per year [6]. Most HAIs must be treated with antibiotics, resulting in increases in antibioticresistant organisms, lengthened hospital stays, additional surgical procedures, inefficiency in hospital systems, disability, and sometimes death.

Careful hand hygiene $(\mathrm{HH})$ performed by healthcare staff is the single most important factor in preventing the transmission of pathogens [7-9]. Research has indicated that up to $50 \%$ of HAIs could be avoided with improvements in $\mathrm{HH}$ compliance $[3,8,9]$. Therefore, $\mathrm{HH}$ is recommended as a routine best practice for all healthcare staff-patient interactions $[3,8]$. Several evidencebased guidelines and interventions have been developed to enhance $\mathrm{HH}$ compliance, $[10,11]$ and most healthcare organizations have invested significant resources in the training and education of their staff. In spite of this, improved compliance with $\mathrm{HH}$ guidelines is difficult to achieve and maintain, partially because education alone does not translate into practice change in the demanding clinical environments in which staff practice [9]. Multiple studies have documented $\mathrm{HH}$ compliance rates to be suboptimal, with a mean observed rate of $40 \%[2,12]$.

To address this concerning lack of professional practice, a variety of promotional strategies and interventions have been studied, yet none have demonstrated significant sustained improvements in $\mathrm{HH}$ compliance [3,12-14]. Multimodal interventions, such as those that include both problem-based and task-oriented $\mathrm{HH}$ education, ongoing $\mathrm{HH}$ audits, and HAI surveillance programs, do improve $\mathrm{HH}$ compliance rates but have failed to be sustainable because they require substantial ongoing costs for educators, auditors, and administrators [13]. Rosenthal et al. [15] reported an atypical improvement in $\mathrm{HH}$ compliance as a result of an education, training, and performance feedback program, with an increase in compliance from $23 \%$ to $65 \%$. The authors claimed a sustained improvement, yet significant ongoing effort in the form of observation twice a week over an 18-month period was used to maintain this change.

Given the current challenges to promote $\mathrm{HH}$, an electronic monitoring system, reminding staff to wash their hands when necessary, may facilitate compliance [16]. Swoboda et al. [17] evaluated an electronic monitoring system using light beams and motion detectors at the threshold of each room, with additional sensors detecting the use of sinks, soap, and alcohol gel dispensers. A voice-prompting system instructed the staff to wash their hands if they had not done so before exiting the room. $\mathrm{HH}$ compliance improved by $41 \%$, and infection rates decreased by $48 \%$; however, the authors concluded that compliance was "at best, fair, even in a population of professionals who understand the importance" (p. 363). No data were collected on staff's responses to being monitored, nor on the acceptability of the system [17].

The limited impact of these described strategies and interventions may be due in part to a failure to identify those factors influencing staff's $\mathrm{HH}$ behavior and subsequent lack of incorporation of these findings into intervention designs and the delivery of the intervention. There is some research available indicating that several barriers and facilitators do influence $\mathrm{HH}$ compliance, $[3,12-14]$ yet this information has not been translated in intervention design and implementation.

\section{A new technology to enhance hand hygiene}

A team of researchers has developed a novel technology that allows electronic $\mathrm{HH}$ monitoring and prompting of staff $[18,19]$. The system consists of individual worn badges that communicate with sensors installed at room entrances and hand wash stations throughout the unit. Staff entering or leaving a room are prompted to wash their hands if they have not done so previously. To the best of the authors' knowledge, no other systems with similar capabilities currently exist. A pilot study exploring the acceptability of this electronic monitoring system (EMS) indicated a favourable response by staff, [20] and a larger study evaluating the efficacy of the system and the sustainability of resulting improvements in $\mathrm{HH}$ compliance has been funded. Nurses on a 50-bed unit will be using the EMS for 12 months, and qualitative and quantitative data will be collected. This novel technology has the potential to improve and sustain compliance beyond what has been accomplished previously [21]. However, as with any intervention aimed at changing professionals' behaviour, its effectiveness is sensitive to context [22,23].

A clear understanding of why staff do or do not change their individual behavior is essential in order to guide intervention design $[22,24]$. More specifically, to justify program implementation of the EMS, it will be of critical importance to understand the perceived barriers and facilitators underlying individual nurses' compliance. Furthermore, this information is crucial to design further interventions, disseminate results, and help governments and others to integrate and update $\mathrm{HH}$ compliance standards and policies [25]. The purpose of this study was therefore to explore barriers and facilitators to current $\mathrm{HH}$ practices and the implementation of the EMS intervention to improve $\mathrm{HH}$ practice from the perspective of 
nurses and administrators. Nurses represent the largest proportion of healthcare staff that have direct contact with patients and are, therefore, an important target group of any $\mathrm{HH}$ intervention. The findings of this study will be used to inform implementation of the EMS.

\section{Methods}

\section{Design}

A qualitative design using semi structured key informant interviews was used to collect data. Key informant interviews consist of qualitative in-depth interviews from a range of people who have firsthand knowledge about the phenomena in the practice setting. These experts, with their particular knowledge and understanding, can provide insight on the nature of problems and give recommendations for solutions. For this specific study, the authors chose to involve the director of care, the unit manager, an infection control specialist, and nurses as key informants.

In determining the approach to gather the information needed, the authors chose the Theoretical Domains Framework (TDF) of behaviour change developed by Michie et al., [26] as the domains aligned well with our aim to explore barriers and facilitators of a $\mathrm{HH}$ behavior change.

\section{Setting and participants}

Semi structured in-depth interviews were undertaken to identify staff's perceived barriers and facilitators to current $\mathrm{HH}$ practices and the implementation of the EMS. All nursing staff employed on the intervention unit were invited to participate in the study. Inclusion criteria included being a part-time or full-time employee on the designated unit and providing direct care. In addition, the infection control specialist and the director of care for the facility and the unit manager responsible for the intervention unit were invited. All potential participants were contacted and received an information letter about the study. If they agreed to receive more information, one of the investigators (VMB) met with them and further explained the study and invited them to participate. Interested employees were then asked to sign a consent form.

Individual interviews were conducted with a convenience sample of three healthcare administrators (one unit manager, one director of care, and one infection control specialist) and eight nurses. There is no firm and fast rule regarding the appropriate sample size in interpretive descriptive research [27]; however, Thorne [28] notes that the vast majority of studies using this approach are likely to be relatively small. In addition, the predetermined designation of the intervention unit limited the sample size; the facility employs only one director of care and three infection control specialists, and the intervention unit employs 24 nurses, further limiting the comparison between nurses' responses and those of the administrators. To address these issues and to determine an appropriate sample size, the authors applied the principles for deciding saturation in theory-based interview studies, outlined by Francis and colleagues [27]. A minimum sample size for initial analysis was determined to be six nurses and three administrators. Subsequently, two more interviews with nurses were conducted without new ideas emerging. The authors were unable to recruit additional administrators, as there were none available.

\section{Ethics}

All recruitment and data collection procedures were approved by the facility's Research Ethics Board prior to the start of the study. Given the potential to identify the individual administrators as the director of care, infection control specialist, or the unit manager, the authors decided to report their data as grouped responses, rather than individual data. No personal information was recorded. Only the researchers had access to the data.

\section{Interview guide}

The TDF of behavior change describes a theoretical domain interview (TDI) [26] with 12 possible domains that can facilitate or hinder successful intervention implementation: knowledge, skills, social/professional role and identity, beliefs about capabilities, beliefs about consequences, motivation and goals, memory and attention and decision processes, environmental context and resources, social influences, emotion, behavioral regulation, and nature of the behaviours (Table 1). All 12 of these domains were of interest, so the TDI guide was adapted to our study. For each of the 12 possible domains that could act as facilitators or barriers to current $\mathrm{HH}$ practices and a successful $\mathrm{HH}$ intervention implementation, the authors developed several interview questions. These questions explored factors that might influence nurses' behavior change related to $\mathrm{HH}$ in general and the specific EMS (Table 1).

\section{Coding reliability}

Each interview followed the same protocol to ensure quality control and started with broad, open-ended questions followed by an increasing focus on specific issues. The interviews were conducted in a private setting using a semistructured guide, audiotaped, and transcribed verbatim. Subsequently, each transcript was reviewed by the interviewer to ensure accuracy and inclusion of expressive detail [29]. 
Table 1 Behavioral-change domains and some interview questions to explore behaviour change

\section{Behavioral-change domains}

Knowledge

Skills

Social/professional role and identity (self-standards)

Beliefs about capabilities (self-efficacy)

Beliefs about consequences (anticipated outcomes/ attitudes)

Motivation and goals (intention)

Memory, attention, and decision processes
Some interview questions to explore behaviour change

Can you describe the guidelines to perform proper $\mathrm{HH}$ ?

Can you discuss when to perform $\mathrm{HH}$ ?

Can you describe why you should be performing $\mathrm{HH}$ ?

Can you describe how the EMS works?

Do you know what information the EMS can collect?

Can you explain the proper procedure of performing $\mathrm{HH}$ ?

How easy or difficult is it to perform $\mathrm{HH}$ on your unit?

Can you describe how to use the EMS?

Do you know how to respond when the EMS reminds you?

What role will the EMS play in enhancing $\mathrm{HH}$ ?

Do you think the EMS should determine how you perform $\mathrm{HH}$ ?

Do you feel that the guidelines for performing $\mathrm{HH}$ with the EMS are congruent with your professional standards of practice?

Should proper $\mathrm{HH}$ be practiced at all levels of staff?

Difficult or easy is it for you to maintain proper $\mathrm{HH}$ ?

What problems have you encountered when trying to practice proper $\mathrm{HH}$ ?

What would help you to increase HH compliance?

How confident are you that you can increase compliance with the barriers and difficulties you face?

How well equipped and comfortable do you feel in increasing your level of HH compliance? When using the EMS?

How capable do you feel in maintaining increased compliance with HH? When using the EMS?

How well will this EMS record your $\mathrm{HH}$ ?

Does HH play an important role in your current practice? For yourself? For your patients? Can you explain why?

Do you believe that this EMS will play an important role in your practice?

Do you foresee any positive or negative outcomes of increased $\mathrm{HH}$ compliance on patient outcomes? Staff outcomes? Do you foresee these outcomes/consequences as long term or short term?

Do you foresee a negative consequence of using the EMS? For patient outcomes? Staff outcomes?

What do you think will happen if $\mathrm{HH}$ compliance is not increased in terms of patient outcomes? Staff outcomes? Do you think these are short- or long-term consequences?

How will you feel if you are able to increase HH compliance? How will you feel if you do not?

Would you like to increase your HH compliance?

Do you feel a need to increase your HH compliance?

What are your reasons for increasing your $\mathrm{HH}$ compliance?

Is there any aspect of your HH performance that you could improve on? Frequency, activity related?

Are there other things that you would like to achieve that might interfere with increasing your $\mathrm{HH}$ compliance?

Are there incentives to increasing $\mathrm{HH}$ compliance? If so, what are they?

Are there incentives to use the EMS? If so, what are they?

Do you usually perform $\mathrm{HH}$ ? How often on a regular shift?

Do you consciously think and make the decision to wash your hands?

What factors influence that decision? Type of care activity? Type of patient? Time?

How much attention do you have to pay to perform $\mathrm{HH}$ ?

Do you remember to perform HH? How?

Do you think the reminder system in the EMS will enhance your $\mathrm{HH}$ ?

Can you think of times where you might not perform $\mathrm{HH}$, such as competing tasks or time constraints? 
Table 1 Behavioral-change domains and some interview questions to explore behaviour change (Continued)

\begin{tabular}{|c|c|}
\hline \multirow{5}{*}{$\begin{array}{l}\text { Environmental context and resources } \\
\text { (environmental constraints) }\end{array}$} & Where do you currently disinfect your hands? \\
\hline & Have you used a wearable alcohol dispenser device? How does this impact your HH performance? \\
\hline & $\begin{array}{l}\text { To what extent do physical or resource factors, such as the availability and functioning of wall units } \\
\text { and technology, facilitate or hinder performing } \mathrm{HH} \text { ? }\end{array}$ \\
\hline & Do you think necessary resources are available for staff to increase HH compliance? \\
\hline & Do you believe that the EMS will enhance your HH performance? \\
\hline \multirow[t]{6}{*}{ Social influences (norms) } & Does HH play an important role on your unit? Can you explain why? \\
\hline & Do you believe that nursing staff on this unit are washing their hands when necessary? \\
\hline & $\begin{array}{l}\text { To what extent do social influences facilitate or hinder performing } \mathrm{HH} \text { ? Social influence from your } \\
\text { peers? Managers? Other professional groups? Patients? Relatives? }\end{array}$ \\
\hline & $\begin{array}{l}\text { Do you believe that there will be social influences from your peers to use the EMS? Managers? } \\
\text { Patients? Other groups? }\end{array}$ \\
\hline & Will you or have you ever observed others performing $\mathrm{HH}$ ? \\
\hline & Do you have role models in performing $\mathrm{HH}$ ? \\
\hline \multirow[t]{3}{*}{ Emotion } & Does performing $\mathrm{HH}$ elicit an emotional response? If so, what? \\
\hline & To what extent will emotional factors facilitate or hinder your $\mathrm{HH}$ ? \\
\hline & Do you believe that emotional factors will influence the use of the EMS? \\
\hline \multirow[t]{3}{*}{ Behavioral regulation } & What initial steps need to be taken to improve HH compliance/use the EMS on an individual level? \\
\hline & How about on an organizational level? \\
\hline & Can you think of any procedures that would encourage increased HH compliance/use of EMS? \\
\hline \multirow[t]{7}{*}{ Nature of the behaviours } & How will the EMS increase HH compliance? \\
\hline & Who needs to work differently for this to occur? When? Where? \\
\hline & How do you know whether increased HH compliance has occurred? \\
\hline & What do you currently do in terms of performing $\mathrm{HH}$ ? \\
\hline & Is this a new or existing behavior that needs to become a habit? \\
\hline & Can the context be used to prompt you to perform HH? (prompts: layout, reminders, equipment) \\
\hline & How long do you think the changes are going to take? \\
\hline
\end{tabular}

$H H=$ hand hygiene; $E M S=$ electronic monitoring system.

\section{Transcript analysis}

Data were extracted by employing an inductive, interactive, comparative process that allows ideas or categories to arise from the data [30]. First, all of the interview data were reviewed and broken down into the TDI theoretical domains. Next, within each theoretical domain, discrete concepts were identified to allow comparisons for similarities and differences (Table 2). An initial coding scheme was developed to permit connections between concepts. The final step consisted of systematically relating core categories to other categories to extract barriers and facilitators that could influence $\mathrm{HH}$ behaviour and the intervention implementation.

Two evaluators independently reviewed interview transcripts to identify key words, phrases, and concepts used by the participants to enhance accuracy of the analysis. Subsequently, they compared and contrasted codes emerging from the data to ensure consistency in the definitions and interpretations of the codes. All data were coded electronically according to standard qualitative coding techniques [28]. NVivo 9 (QSR International,
Cambridge, MA, USA), an advanced storage-code-andretrieval software program, facilitated the organization and analysis of the data. To ensure the reliability of the analyses, an audit trail was used to maintain records and encourage reflexivity of data collection and analysis procedures [31].

\section{Results}

Participants had no difficulty commenting on each of the different theoretical domains. The data provide valuable insights into our understanding of potential barriers and facilitators within each domain to current $\mathrm{HH}$ practices and to implementing the EMS intervention in clinical practice. A summary of the key findings is presented below.

\section{Knowledge}

The knowledge domain contains the information the nurses have in regards to $\mathrm{HH}$, the rationale about $\mathrm{HH}$ compliance, the scientific evidence supporting $\mathrm{HH}$, and the procedural knowledge to perform $\mathrm{HH}$ and explores 
Table 2 Behavioral-change domains and descriptive codes

\begin{tabular}{|c|c|}
\hline Behavioral-change domains & Descriptive codes \\
\hline Knowledge & Description of information and knowledge as facilitators to performing $\mathrm{HH}$ and using the EMS. \\
\hline Skills & Description of skills and competencies as facilitators to performing $\mathrm{HH}$ and using the EMS. \\
\hline $\begin{array}{l}\text { Social/professional role and identity } \\
\text { (self-standards) }\end{array}$ & $\begin{array}{l}\text { Description of the staff's role and identity on the unit as facilitators to performing } \mathrm{HH} \text { and using } \\
\text { the EMS. }\end{array}$ \\
\hline Beliefs about capabilities (self-efficacy) & $\begin{array}{l}\text { Description of perceived self-efficacy and control of behaviors as facilitators to performing } \mathrm{HH} \text { and } \\
\text { using the EMS. }\end{array}$ \\
\hline $\begin{array}{l}\text { Beliefs about consequences (anticipated } \\
\text { outcomes/ attitudes) }\end{array}$ & Description of beliefs about the consequences as facilitators to performing $\mathrm{HH}$ and using the EMS. \\
\hline Motivation and goals (intention) & Description of motivation and goals as facilitators to performing $\mathrm{HH}$ and using the EMS. \\
\hline Memory, attention, and decision processes & $\begin{array}{l}\text { Description of memory, attention, and decision processes as facilitators to performing } \mathrm{HH} \text { and using } \\
\text { the EMS. }\end{array}$ \\
\hline $\begin{array}{l}\text { Environmental context and resources } \\
\text { (environmental constraints) }\end{array}$ & $\begin{array}{l}\text { Description of availability and accessibility of resources as facilitators to performing } \mathrm{HH} \text { and using the } \\
\text { EMS. }\end{array}$ \\
\hline Social influences (norms) & $\begin{array}{l}\text { Description of social influence and the role of the healthcare team as facilitators to performing } \mathrm{HH} \\
\text { and using the EMS. }\end{array}$ \\
\hline Emotion & $\begin{array}{l}\text { Description of emotion (stress, fear, burnout, or positive or negative emotional responses) as a facilitator } \\
\text { to performing } \mathrm{HH} \text { and using the EMS. }\end{array}$ \\
\hline Behavioral regulation & Description of behavioral regulation as a facilitator to performing $\mathrm{HH}$ and using the EMS. \\
\hline Nature of the behaviours & Description of the nature of the behavior as a facilitator to performing $\mathrm{HH}$ and using the EMS. \\
\hline
\end{tabular}

$H H=$ hand hygiene; $E M S=$ electronic monitoring system.

if these act as facilitators with regards to $\mathrm{HH}$. This domain also includes the participants' understanding and perceived rationale behind the EMS system, which could potentially facilitate the intervention. Our main finding for this domain was that the administrators (ADM), consisting of the director of care, the unit manager, and the infection control specialist, all believed that the staff have some understanding and knowledge relevant to the implementation of the EMS, yet this knowledge is not sufficient. Nurses, on the other hand, were confident they have the required knowledge to perform $\mathrm{HH}$ and use the EMS. Examples of this included the following:

"I would assume that people that work within our program should be well advised." (ADM 1)

"We have enough education, we also have education from infection control, they come up and do in-services, they also have it on the computer and also give handouts." (RN 1, Interview 3)

\section{Skills}

This domain describes the skills and competencies to perform $\mathrm{HH}$ and the procedural ability to use the EMS. Again, the data reveal a strong representation of this domain by nurse participants who stated that they have the skills required for performing $\mathrm{HH}$ and using the system.

"It's not difficult at all. You have to use sanitizer or water and soap to wash your hands. No, not difficult at all, because we are always having in-services and computerized Q \& A that we do from time to time and other sort of information around."

(RN 2, Interview 4)

Administrators however, perceived the skill level of the nurses as a potential limitation. The following quote demonstrates this discrepancy:

"Yes, I think they have some of those skills. It is just hard to focus on it." (ADM 1)

\section{Social/professional role and identity}

The third domain describes the nurses' role and identity in regards to performing $\mathrm{HH}$ and the EMS system. Nurses and administrators clearly understood the purpose of the system and thought positively about the credibility of the EMS. Both groups describe the system as supporting nurses with their professional identity. The social and group norms are demonstrated by these quotes:

"This [the electronic monitoring system] is going to be very helpful, we have to focus it correctly and to the organization." (ADM 2)

"I think we will be more aware and more mindful of stopping and taking the time to hand washing before we move on, and when we come in and out of the room. It is kind of drilled in our head now." (RN 3, Interview 5) 


\section{Beliefs about capabilities}

This domain describes nurses' perceived self-efficacy and control as potential barriers or facilitators to perform $\mathrm{HH}$ and use the EMS:

"I think it will increase $[\mathrm{HH}]$. . it will prompt us to perform $\mathrm{HH}$ more differently along with the education we get. I think it will lead us in the right direction." (RN 3, Interview 5)

"Part of providing safe care, which is one of the standards, is to make sure that your hands are clean when you go to the patient." (RN 2, Interview 4)

Administrators accordingly described the nurses' beliefs about capabilities, yet added some difficulties within the healthcare environment that could potentially interfere with the use of the system. In other words, both groups perceived nurses to have behavioral control, yet administrators listed external difficulties and barriers that would limit nurses' ability to perform proper $\mathrm{HH}$ :

"I am a great believer that staff can perform at a very high level but unfortunately, there are other things that come up in that particular moment that may take their mind away from doing what they know is best practice." (ADM 2)

\section{Beliefs about consequences}

The next domain describes nurses' beliefs about the consequences of $\mathrm{HH}$ and using the EMS as potential barriers or facilitators. In the interviews, nurses focused consistently on the immediate outcome, that is, responding to the reminder signal to perform $\mathrm{HH}$.

"I think [the EMS] will be very helpful and it will be a good reminder, make people aware of how often they wash their hands and the importance of washing their hands." (RN 1, Interview 3)

"It will impact everyone and it's going to be $\mathrm{HH}$ on a higher level." (RN 5, Interview 7)

In contrast, administrators did not discuss the immediate consequences of the EMS but talked about the longitudinal aspect of the EMS data and the subsequent evaluative component for comparing nurses' performance. Administrators also described the contingencies of the EMS, the benefits of using the system outweighing the costs, and the reinforcement and/or rewards when nurses did not perform as expected.

"I think with any system like this, at the beginning, it is going to be very confidential and perhaps down the line it may be reported to managers, and that is always a concern for the staff." (ADM 3)
Only the administrators discussed the cost associated with increased $\mathrm{HH}$ compliance and the effect of the system that might lead to poor implementation of the EMS.

\section{Motivation and goals}

This domain explores the barriers or facilitators to the motivations and goals to practice $\mathrm{HH}$ and use the EMS system. This domain refers to how high a priority the target behaviour is for the participant. Nurses concentrated foremost on their personal safety and their families' safety as a source of motivation to perform $\mathrm{HH}$ :

"Since we had SARS, people are very, very conscious about what they can take home to their family, so they are very, very conscious." (RN 1, Interview 3)

Interestingly, only one of the nurses mentioned patient safety when discussing the intention to perform $\mathrm{HH}$ or use the system initially. Administrators, on the other hand, did not discuss nurses' personal safety as a motivator but believed that nurses would display some intrinsic and external motivators to perform $\mathrm{HH}$, such as professional commitment, individual and unit incentives, and personalized goal setting:

"Incentive is good for a group who want to do well. There is no incentive for the others who draw back and do nothing, depends on the individual and the group." (ADM 3)

"... even with results from infection control, it is always shared with staff and they are proud to hear what the results are and it's just going to be ongoing with continuous feedback and enhance the hand hygiene." (ADM 2)

The emphasis on a different characteristic within the domain of motivation and goals suggests that the implementation of the EMS will need to address these differing perspectives.

\section{Memory, attention, and decision processes}

This domain explores memory, attention, and decision processes as barriers or facilitators in regards to influencing nurses' decisions to wash hands and use the EMS system. As optimal $\mathrm{HH}$ is considered best practice in nursing practice, it would be assumed that nurses perceive $\mathrm{HH}$ as a routine procedure, a task they usually carry out before entering or after leaving a patient room. Nurses supported this assumption by clearly stating that they are constantly aware of the need to wash their hands, as demonstrated by these quotes:

"I am consciously thinking about [performing $\mathrm{HH}$ ], especially when you are in a room with four 
people...we don't want to go back and forth and you try to be more aware, or when you are coming out of the room, you try to make sure you wash before going in." (RN 3, Interview 5)

"...I see the pump right there in the hall, so once I see it, I use it and go into the room and when I come out, I look for it and use it again ...." (RN 3, Interview 5)

Conversely, administrators stated that the staff do not remember to focus on performing $\mathrm{HH}$ and do not have the decision processes in place to judge whether $\mathrm{HH}$ is necessary or not.

"I think they don't have time and I think they don't regard it seriously." (ADM 3)

This interesting disparity calls for a need to collect data on the actual attention and decision processes of the nurses during the intervention implementation of the EMS.

\section{Environmental context and resources}

This domain explores the availability and accessibility of resources to perform $\mathrm{HH}$ and use the EMS system as a facilitator to enhance $\mathrm{HH}$ and the EMS intervention. Both administrators and nursing staff felt there were adequate resources available, and accessibility and functionality of the resources were not deemed problematic.

"When I worked in long-term care there were no sinks in any of the rooms and now, you can't go far out of a room without finding one." (ADM 3)

"I think there are enough [resources] that are in place where we can easily access it." (RN 3, Interview 5)

The above quotes indicate that this domain is relevant, yet not problematic, to the implementation of the EMS.

\section{Social influences}

This next domain explores social influence as a potential barrier or facilitator in improving $\mathrm{HH}$ and using the EMS. Nurses and administrators had a very different perspective on the relevance of social influence. Administrators assumed that social support by peers, administrators, other professional groups, or patients would facilitate $\mathrm{HH}$ compliance. Furthermore, they anticipated that strong performers would act as role models and leaders to invite weaker performers to increase their $\mathrm{HH}$ performance. Teamwork and group cohesion will be a strong support to enhance the implementation of the EMS. Administrators also perceived that social support and teamwork would be utilized in a positive manner to help everyone achieve the goal of enhanced $\mathrm{HH}$.
"I think that there are some staff that really get it and there could be a peer support system." (ADM 3)

"When you have a role model that already thinks so positively of them [nurses], then they want to follow that type of role model." (ADM 2)

Conversely, nurses described the social influences as a deterrent to implementing the system. Nurses were not interested in group conformity or social comparisons. Rather, they focused on their own behavior as negative social influence, potentially causing inter-group conflict. Nurses were focused on improving their individual performance and did not want to act as role models or leaders. They would rather leave poor performers to their own devices.

“. . people do watch other people.... people would not like to talk or work with other people that did not wash their hands, so they are going to shun that person." (RN 1, Interview 3)

"... once you say something you make them feel like you are watching them, looking for some mistakes, and of course, they're going to retaliate. They're going to do the same thing to you. And then it's just going to escalate and then you're going to have problems. It is good to tell them, I understand that, but there are consequences." (RN 6, Interview 9)

\section{Emotion}

The domain of emotion delves into any stress, fear, burnout, or emotional responses (positive or negative) that could be caused by performing $\mathrm{HH}$ and using the EMS acting as potential barriers or facilitators to enhancing $\mathrm{HH}$. The authors note that this domain might appear to overlapping with the domain on the beliefs about consequences. To avoid any confusion, the authors strictly adhered to the theoretical domains discussed in the interviews and reported the results as such.

The EMS records or prompts nurses to comply with each opportunity for $\mathrm{HH}$. Given a current $\mathrm{HH}$ compliance rate of below $40 \%$, it is anticipated that nurses will have to double their $\mathrm{HH}$ activity, potentially causing a cognitive overload or even physical tiredness. The administrators seemed to be well aware of this risk, claiming that some of the $\mathrm{HH}$ performance measures cause frustration or a negative emotional response in staff:

"Guidelines could drive them crazy." (ADM 2)

Nurses described negative emotions in relation to their own and families' safety, although this was not directed towards the EMS or the $\mathrm{HH}$ practice. 
"Fear and safety, you know because when we are here and we are more prone to anything that is here, then you don't want to bring home flu to anyone else."

(RN 3, Interview 5)

\section{Behavioral regulation}

This domain describes the behavioral regulation, that is, the preparatory steps needed to perform enhanced $\mathrm{HH}$ and use the EMS, as a barrier or facilitator. Within this domain, nurses described the importance of individual feedback and self-monitoring in order to increase their performance. Nurses reported being in favor of meeting with a mentor on a one-to-one basis to discuss goals and targets and to identify priorities and analyze performance data to support improvement:

"At the individual level, reminders. I think at the organizational level we are not doing too bad because we are doing a lot of teaching, so you know, it remains ongoing. We will get to a point where it will be at a higher level." (RN 3, Interview 5)

Administrators however, believe that this behavioral regulation might be a barrier in the implementation of the EMS. As one of the administrators describes,

"I don't think they [nurses] understand the importance. I think it still goes back to the education and we don't have a lot of infections here so, why should I bother to get it? They also haven't had patients ask them, 'Have you washed your hands before you touch me?”' (ADM 1)

\section{Nature of behaviours}

This last domain depicts the nature of the proposed behaviour, that is, improved $\mathrm{HH}$ compliance, as a facilitator in regards to the current $\mathrm{HH}$ practice and the use of the EMS system. According to nurses, $\mathrm{HH}$ is currently being practiced as a routine, relying on habit. Yet, research also indicates that this "routine" is followed in less than half of the situations where $\mathrm{HH}$ actions are required. The proposed $\mathrm{HH}$ behavior will not be any different than the current practice, however, the behavior will need to be performed more frequently. Nurses were aware that, as a group, the existing behavior needed to be practiced more often and that the EMS will prompt this new behavior:

"It maybe varies with other team members, yes, but with nursing, I think it is more on the table to keep your hands clean when going to another patient. Take your glove off and wash your hands. And I think it is because of all the education sessions, reminders like the [EMS]." (RN 1, Interview 3)
Administrators supported this response as well but indicated that increasing the frequency of the proposed behavior might be more difficult than anticipated:

". . you have to build in sustainability and accountability, right. . And the accountability can't always lie with the manager. The staff has to be accountable, but how are we going to monitor it to make sure it is sustained? Because it falls off real quick." (ADM 3)

\section{Discussion}

This study used the TDI [26] to explore barriers and facilitators to current $\mathrm{HH}$ practices and the implementation of a new EMS to improve $\mathrm{HH}$ practice. Semistructured in-depth interviews were conducted that compared perceptions of nurses and administrators. In this study, facilitators and barriers to current $\mathrm{HH}$ practices and the implementation of the EMS intervention ranged from the individual level to organizational and social contexts. For the majority of the theoretical domains, differences in responses were noted between the administrators and the nurses. These differences provide insight into considerations for how the intervention should be implemented, as directed by the knowledge-to-action cycle [22].

Knowledge was perceived both as a barrier and facilitator by the participants. Nurses believed they already had sufficient knowledge about $\mathrm{HH}$, whereas the administrators perceived that the nurses did not; therefore, the lack of knowledge acted as a barrier to current $\mathrm{HH}$ practices and the implementation of the EMS. This discrepancy will need to be addressed in the implementation plan for the EMS intervention. If nurses believe they have the knowledge and awareness as to how and when to perform $\mathrm{HH}$, competency testing and educational sessions to update nurses' knowledge and skills will not be viewed as useful. Rather, a focus on understanding why nurses decide to perform or skip $\mathrm{HH}$ actions may be of greater importance for the successful implementation of the EMS. For example, some of these reasons to perform or neglect $\mathrm{HH}$ could be explored during weekly and monthly educational sessions with the participants, rather than just focusing on a discussion of actual $\mathrm{HH}$ compliance rates [18].

One of the biggest discrepancies between the two groups was related to the beliefs about consequences domain. Administrators clearly indicated the potential consequences of using the EMS in the long term as a potential barrier, whereas nursing staff only perceived the immediate outcomes of the system. The lack of nurses' focus on long-term outcomes might indicate that nurses do not realize the capabilities of the system, a problem easily corrected by providing additional information in the recruitment session or during the individualized 
educational sessions [18]; it might also point to a more serious issue based in a lack of ongoing commitment to improve practice. This phenomenon has been reported by other researchers analyzing quality improvements in nursing practice [32] and results from the current practice development movement focusing on immediate reportable outcomes and the lack of a systematic approach to the practice change. As a result, nurses act upon the immediate demand to change practice, yet they do not perceive the change in practice to be necessary nor valuable [32]. In order to create a sustainable $\mathrm{HH}$ practice change, it will be necessary to continuously explore and address individual nurses' development and empowerment during the intervention phase.

Another major difference was in the social influences domain. Nurses did not describe social influences, such as the opinion or care practice of colleagues, as an enabler to implementation of the EMS. Nurses were focused on improving their individual performance and preferred to stay away from poor performers as opposed to creating a supportive network to enhance $\mathrm{HH}$ practice. The individuality of nursing performance has previously been described in the nursing literature [33]. Despite careful evidence on appropriate levels of $\mathrm{HH}$ and staff performance, $[11,33]$ it appears that this knowledge was not translated to staff and administrators in this practice setting. Both administrators and nursing staff in this study and others did not know how often staff perform $\mathrm{HH}$ or the level of performance at the unit or facility level, nor do they know the acceptable $\mathrm{HH}$ compliance rates [34]. When developing the implementation plan, the integration of anonymous group data in the individual performance reports and the discussion of these data in the educational sessions with the individual participants might create a heightened awareness of one's own performance compared to others [18,22,35]. The apparent differences between administrators and nurses in regards to several barriers and facilitators toward $\mathrm{HH}$ points to an intervention implementation strategy that will need to focus on the provision of support and mentoring for the nurses, rather than solely improving the frequency of $\mathrm{HH}[18,35]$.

Based on the data, the next step in the EMS intervention implementation needs to consist of identifying behavior-change techniques that target these specific determinants, such as self-monitoring of performance, graded goal setting and improving $\mathrm{HH}$ practice, and modeling and demonstration of behaviors by others [20-22,35]. Norms can be addressed by modeling/ demonstration of behaviors by others; social processes of encouragement, pressure, and support; and prompts, triggers, cues [35,36]. Another behavior-change technique that will be considered during the intervention implementation is the use of the EMS data to create "social influences" in order to enhance the uptake [36]. Weekly individual performance data reports will include a graph that indicates other participants' overall compliance data to compare personal data against the levels in the group. During individual meetings with the participant, specific information on how to increase $\mathrm{HH}$, various techniques for increasing $\mathrm{HH}$ (prompts, triggers, cues), and suggestions on how to change the behavior will be provided [18].

Limitations identified in this research include potential social desirability bias, the use of a simple coding scheme, and the small sample from one hospital unit. Further research is required to validate and refine this theoretical framework and the coding procedure [24]. Specific areas of interest for this research are related to identifying the most important determinants or behaviourchange domains, so as to guide the selection of which determinants to address and/or which of the techniques to adopt when implementing an intervention. It would also be beneficial to explore the relationship between the domains. If the awareness or skills are improved, would this change affect beliefs or capabilities? Lastly, it is important to note that despite the overall agreement within the nurses' sample, the responses represent perceptions and not actual skills or knowledge. This was clearly demonstrated when all nurses claimed they had sufficient knowledge of $\mathrm{HH}$ guidelines, yet there was a clear gap in $\mathrm{HH}$ knowledge when asked specifically about $\mathrm{HH}$ skills. This suggests that relying on only perceptions is not sufficient and could negatively impact the implementation of the intervention.

\section{Significance}

HAIs affect many patients and often result in suffering and deaths. Consistent $\mathrm{HH}$ can reduce these infection rates by up to $50 \%$, yet healthcare staff often fail to perform $\mathrm{HH}$. Several attempts have been made to improve compliance, without significant sustained increases. Novel approaches combined with a clear understanding of how to change nurses' behaviour are required to improve $\mathrm{HH}$ compliance.

This work is an essential first step towards identifying factors affecting nurses' behavior change associated with $\mathrm{HH}$ compliance. Findings of this study will guide the identification of optimal strategies to enhance adoption of "best $\mathrm{HH}$ practice" by nurses working in different practice environments and will inform the subsequent intervention study [22]. The end goal of the intervention study is to enhance and sustain improved $\mathrm{HH}$ compliance and, ultimately, improve the safety and health of patients receiving care.

Findings of this study will lead to a better understanding of the effect of an intervention employing a monitoring system on $\mathrm{HH}$ compliance and will be instrumental 
in providing evidence to support intervention design and interpretation and decisions made by policy makers around $\mathrm{HH}$ practices. By enhancing and understanding factors underlying $\mathrm{HH}$ behaviour, this research will ultimately contribute to safer and better-quality care for patients. This study also contributes to the knowledge translation literature in that the knowledge generated from this work will provide a structured approach to developing an implementation plan with a better understanding of how to enhance healthcare professionals' behaviours in complex interventions.

\section{Conclusion}

This qualitative study demonstrated the value in using psychological theories commonly used in knowledge translation to explore barriers and facilitators to current $\mathrm{HH}$ practices and when implementing an intervention to improve $\mathrm{HH}$ practice. Perspectives of administrators and nurses were compared for their relevance to $\mathrm{HH}$ and the implementation of an EMS to enhance $\mathrm{HH}$. The results provide a better understanding of nurses' behaviourchange processes of the EMS intervention and will be integrated to refine the implementation plan.

Rigorous exploration of theoretical domains of behavior change acting as potential barriers and facilitators will not only lead to appropriate selection of the intervention components but will also promote the adaptation of the knowledge to the local context. In respecting these simple, yet essential, steps in the process of integrating knowledge into practice before actual intervention design and implementation, it is hypothesized that interventions will be more successful and acquired knowledge more sustainable.

\section{Competing interests}

VMB received funding from Toronto Rehabilitation Institute for the development and testing of the EMS. GRF is Vice President of Research at Toronto Rehabilitation Institute and is the principal investigator on the EMS project. The other authors declare they have no competing interests.

\section{Authors' contributions}

VMB was responsible for the research idea and project management and led the interview design and data analysis. She wrote the first draft of the paper and subsequent redrafts. All authors contributed to the development of the research objectives and methods and to the writing of the paper. JHL supported the data collection and helped analyze data. All authors read and approved the final research protocol and manuscript. All authors contributed equally to this study.

\section{Authors' information \\ VMB is currently the CIHR Schlegel Chair for Enhanced Seniors' Care, Schlegel-University of Waterloo Research Institute for Aging (RIA) \& School of Health \& Life Sciences and Community Services, Conestoga College Institute of Technology and Advanced Learning. At the time of the study, VMB was a postdoctoral fellow at Toronto Rehabilitation Institute. SBJ is the Toronto Rehabilitation Institute Chair at the University of Toronto.}

\section{Acknowledgments}

Funding for this study was received by the Canadian Institute of Health Research (funding reference \#HTX 80047, XHT 83447, and CIHR 102887), Toronto Rehabilitation Institute, and the Ontario Centers of Excellence
(Project \# TO-CR-1007 4-08). Toronto Rehabilitation Institute receives funding under the Provincial Rehabilitation Research Program from the Ministry of Health and Long-Term Care in Ontario.

\section{Author details}

${ }^{1}$ Schlegel-University of Waterloo Research Institute for Aging (RIA) \& School of Health \& Life Sciences and Community Services, Conestoga College Institute of Technology and Advanced Learning, Kitchener, ON, Canada. ${ }^{2}$ Toronto Rehabilitation Institute, Toronto, ON, Canada. ${ }^{3}$ Department of Surgery, University of Toronto, Toronto, ON, Canada. ${ }^{4}$ Department of Physical Therapy, University of Toronto, Toronto, ON, Canada.

Received: 3 October 2011 Accepted: 8 August 2012

Published: 28 August 2012

\section{References}

1. Daschner FD: The transmission of infections in hospitals by staff carriers, methods of prevention and control. Infect Control 1985, 6(3):97-99.

2. Haas JP, Larson EL: Compliance with hand hygiene guidelines: Where are we in 2008? Am J Nurs 2008, 108(8):40-44.

3. World Health Organization: WHO Guidelines on Hand Hygiene in Healthcare. Geneva: WHO; 2009.

4. Centers for Disease Control (CDC): Public health focus: surveillance, prevention, and control of nosocomial infections. MMWR Morb Mortal Wkly Rep 1992, 41(42):783-787.

5. Stone PW, Larson E, Kawar LN: A systematic audit of economic evidence linking nosocomial infections and infection control interventions: 1990-2000. Am J Infect Control 2002, 30(3):145-152.

6. Zoutman DE, Ford BD, Bryce $\mathrm{E}$, et al: The state of infection surveillance and control in Canadian acute care hospitals. Am J Infect Control 2003, 31(5):266-272.

7. World Health Organization (WHO): WHO Guidelines on Hand Hygiene in Health Care (Advanced Draft). Geneva: World Health Organization; 2006.

8. Health Canada Laboratory Centre for Disease Control, Division of Nosocomial and Occupational Infections: Construction-related nosocomial infections in patients in health care facilities, Canada Communicable Disease Report. Ottawa, Canada: Health Canada; 2011.

9. Pittet D, Hugonnet $\mathrm{S}$, Harbarth $\mathrm{S}$, et al: Effectiveness of a hospital-wide programme to improve compliance with hand hygiene. Lancet 2000, 356(9248):2196-2205.

10. Stone SP: Hand hygiene - the case for evidence-based education. J R SOC Med 2001, 94(6):278-281

11. Boyce JM, Pittet $D$, Healthcare Infection Control Practices Advisory Committee, \& HICPAC/SHEA/APIC/IDSA Hand Hygiene Task Force: Guideline for hand hygiene in health-care settings. recommendations of the healthcare infection control practices advisory committee and the HICPAC/SHEA/APIC/IDSA Hand hygiene task force. Morb Mortal Wkly Rep Recomm Rep 2002, 51(RR16):1-44.

12. Gould D, Chudleigh JH, Moralejo D, Drey N: Interventions to improve hand hygiene compliance in patient care. Cochrane Database Syst Rev 2007, 2:1-18.

13. Naikoba S, Hayward A: The effectiveness of interventions aimed at increasing handwashing in healthcare workers - a systematic review. J Hosp Infect 2001, 47(3):173-180.

14. Rosenthal VD, Guzman S, Safdar N: Reduction in nosocomial infection with improved hand hygiene in intensive care units of a tertiary care hospital in Argentina. Am J Infect Control 2005, 33(7):392-397.

15. Boscart VM, Gorski S, Holliday P, Hufton G, Levchenko A, Marquez-Chin C, McGilton K, Momen K, Tsang M, Fernie G: Advanced technologies to curb healthcare-associated infections. HealthcarePapers 2009, 9(3):52-55.

16. Swoboda SM, Earsing K, Strauss K, Lane S, Lipsett PA: Electronic monitoring and voice prompts improve hand hygiene and decrease nosocomial infections in an intermediate care unit. Crit Care Med 2004, 32(2):358-363

17. Boscart VM, Levchenko Al, Fernie GR: Defining the configuration of a hand hygiene monitoring system. Am J Infect Control 2010, 38(7):518-522.

18. Levchenko Al, Hufton GC, Boscart VM, Fernie GR: Embedded system for hygiene compliance monitoring. IEEE Transactions on Automation Science and Engineering 2010, 7(3):695-698. 
19. Boscart VM, McGilton K, Levchenko A, Hufton G, Holliday P, Fernie G Acceptability of a wearable hand wash device with monitoring capabilities. Journal of Hospital Infections 2008, 70:216-222.

20. Boscart VM, Levchenko A, Fernie G: Automated hand hygiene monitoring: Perspectives for healthcare staff, management, and infection control specialists. ICU Management 2010, 10(1):28-30

21. Straus S, Tetroe J, Graham ID: Knowledge translation in health care: Moving from evidence to practice. 1st edition. Oxford: Blackwell Publishing Ltd; 2009.

22. Grol R: Success and failures in the implementation of evidence-based guidelines for clinical practice. Medical Care 2001, 39:1146-1154.

23. Michie S, Berentson-Shaw J, Pilling S, Feder G, Dieppe P, Raine R, Cluzeau F, Alderson $P$, Ellis S: Turning evidence into recommendations: protocol of a study guideline development groups. Implementation Science 2007, 5(2):29.

24. Health Canada LCfDCDoNaOl: Routine practices and additional precautions for preventing the transmission of infection in health care. Can Commun Dis Rep 1999, 25(S4):1-142.

25. Michie S, Johnston M, Abraham C, Lawton R, Parker D, Walker A: Making psychological theory useful for implementing evidence based practice: a consensus approach. Qual Safe Health Care 2005, 14:26-33.

26. Thorne S: Interpretive description. Walnut Creek, CA: Left Coast Press; 2008.

27. Francis JJ, Johnston M, Robertson C, Glidewell L, Entwistle V, Eccles MP, Grimshaw JM: What is an adequate sample size? Operationalising data saturation for theory-based interview studies. Psychol Health 2010, 25(10):1229-1245.

28. Poland B: Transcription quality as an aspect of rigor in qualitative research. Qualitative Inquiry 1995, 1(3):290-310.

29. Glesne C, Peshkin A: Becoming qualitative researchers: An introduction. White Plains, NY: Longman; 1992.

30. Schwandt T: Dictionary of qualitative inquiry. 2nd edition. Thousand Oaks, CA: Sage Publications; 2001

31. Masters K: Role development in professional nursing practice. Sudbury, MA: Jones \& Bartlett Publishers, Inc; 2005.

32. Melnyk BM, Fineout-Overholt E, Stone P, Ackerman M: Evidence-based practice: The past, the present, and recommendations for the millennium. Paediatr Nurs 2000, 26(1):77-80.

33. Muller MP, Detsky AS: Public reporting of hospital hand hygiene compliance - helpful or harmful? JAMA 2010, 304(10):1116-1117.

34. Michie S, Johnston M, Francis J, Hardeman W, Eccles M: From theory to intervention: Mapping theoretically derived behavioural determinants to behaviour change techniques. Applied Psychology: An International Review 2008, 57(4):660-680.

35. Abraham C, Michie S: A taxonomy of behavior change techniques used in interventions. Health Psychol 2008, 27(3):379-387.

36. Janzen AJ, Silvius J, Jacobs S, Slaughter S, Dalziel W, Drummond N: What is a health expectation? Developing a pragmatic conceptual model from psychological theory. Health Expect 2006, 9(1):37-48.

doi:10.1186/1748-5908-7-77

Cite this article as: Boscart et al:: Using psychological theory to inform methods to optimize the implementation of a hand hygiene intervention. Implementation Science 2012 7:77.

\section{Submit your next manuscript to BioMed Central and take full advantage of:}

- Convenient online submission

- Thorough peer review

- No space constraints or color figure charges

- Immediate publication on acceptance

- Inclusion in PubMed, CAS, Scopus and Google Scholar

- Research which is freely available for redistribution 